\title{
How much income inequality is fair? Nash bargaining solution and its connection to entropy
}

\author{
Venkat Venkatasubramanian ${ }^{\mathrm{a}, *}, \mathrm{Yu} \mathrm{Luo}^{\mathrm{b}}$ \\ ${ }^{a}$ Department of Chemical Engineering, Columbia University, New York, NY 10027 \\ ${ }^{b}$ Department of Chemical and Biomolecular Engineering, University of Delaware, Newark, \\ DE 19716
}

\begin{abstract}
The question about fair income inequality has been an important open question in economics and in political philosophy for over two centuries with only qualitative answers such as the ones suggested by Rawls, Nozick, and Dworkin. We provided a quantitative answer recently, for an ideal free-market society, by developing a game-theoretic framework that proved that the ideal inequality is a lognormal distribution of income at equilibrium. In this paper, we develop another approach, using the Nash bargaining solution (NBS) framework, which also leads to the same conclusion. Even though the conclusion is the same, the new approach, however, reveals the true nature of NBS, which has been of considerable interest for several decades. Economists have wondered about the economic meaning or purpose of the NBS. While some have alluded to its fairness property, we show more conclusively that it is all about fairness. Since the essence of entropy is also fairness, we see an interesting connection between the Nash product and entropy for a large population of rational economic agents.

\footnotetext{
${ }^{*}$ Corresponding author

Email addresses: venkat@columbia.edu (Venkat Venkatasubramanian), yuluo@udel.edu (Yu Luo)
}

Preprint submitted to Physica A: Statistical Mechanics and its Applications July 24, 2018 


\section{Introduction}

Extreme economic inequality is widely seen as a serious concern to the future of stable and vibrant capitalist democracies. In 2015, the World Economic Forum in Davos identified deepening income inequality as the number one challenge of our time. As many political observers remarked, the social and political consequences of extreme economic inequality and the uneven sharing of prosperity seem to have played a role in the outcome of the U.S. presidential election in 2016. Many in the U.S. feel that the nation's current level of economic inequality is unfair and that capitalism is not working for $90 \%$ of the population $[1,2,3]$.

Yet some income inequality is inevitable, even desirable and necessary for a successful capitalist society. As different people have different talents and skills, and different capacities for work, they make different contributions in a society, some more, others less. Therefore, it is only fair that those who contribute more earn more. But how much more? In other words, what is the fairest inequality of income? This critical question is at the heart of the inequality debate. The debate is not so much about inequality per se as it is about fairness.

Consider a simple example to illustrate this point. John is hired as a temporary worker to perform a job for one hour and makes $\$ 100$. Lilly also performs the same job, at the same level of quality, but works for two hours and makes $\$ 200$. Is there inequality in their incomes? Of course, there is. But is the inequality fair? Of course, it is. Lilly earned more because she contributed more. Their incomes are not equal, but equitable. They are both paid at the same rate per hour of work, which is the basis for fairness here.

In this simple case, it was easy to ensure equity, but how do we accomplish this, in general, in a free market society consisting of millions of workers of varying degrees of talent, skill, and capacity for work? Is there a measure of fairness that can guide us to accomplish this? Is there an income distribution that ensures equity? Given the complexity of the problem, one might anticipate the answer to be no for both questions. But, surprisingly, the answer is yes, under certain conditions. 
The first author has shown, in a series of papers $[4,5,6]$ and a book [7], that the measure of fairness we are seeking is entropy and the equitable income distribution is lognormal at equilibrium in an ideal free market. These results were arrived at by analyzing the problem in two different, but related, perspectives: statistical mechanics and potential game theory.

In this paper, we demonstrate yet another approach, namely, the Nash bargaining solution (NBS) formalism $[8,9]$. We consider this paper to be valuable in two respects. One, this is the first time one has proposed the NBS formalism for the income distribution problem. Even though the NBS formalism has been well-known for nearly seventy years, and has been used extensively in many fair allocation problems (see, for example, [9]), we find it quite surprising that it has not been used to address the central question of fair distribution of income in a free-market economy. Thus, we consider our NBS formulation to this problem as an important contribution to economic literature. Secondly, while the final result of our analysis in itself is not new, this new approach, however, reveals something unexpected, namely, the true meaning of the Nash product and its connection with entropy.

In the NBS formalism, one arrives at the solution by maximizing the product of utilities, known as the Nash product (more on this in Section 4). Over the years, economists have wondered about the true meaning, i.e., about the economic content, of the Nash product. The sum of utilities of different agents makes economic sense as it gives us the total utility of the system, but why a product of utilities? What does it stand for?

As Trockel [10] observed:

While all other characterizations, via axioms or via support by equilibria of non-cooperative games appear to reflect different aspects of this solution and to open new ways of interpretation, its most simple description via the Nash product seems to have escaped up to now a meaningful interpretation ... Yet, concerning its direct interpretation the situation is best described by the quotation of Osborne 
and Rubinstein [11] (1994, p. 303): "Although the maximization of a product of utilities is a simple mathematical operation it lacks a straightforward interpretation; we view it simply as a technical device."

Most people use it as a convenient mathematical device, but what is it really? Interestingly, the meaning of potential $\left(P^{*}\right)$ in game theory also had posed a similar puzzle as Monderer and Shapley [12] had pointed out:

This raises the natural question about the economic content (or interpretation) of $P^{*}$ : What do the firms try to jointly maximize? We do not have an answer to this question.

This is again related to a similar fundamental question raised by Samuelson [13] decades ago in his Nobel lecture:

What it is that Adam Smith's "invisible hand" is supposed to be maximizing?

We showed in our earlier work $[6,7]$ that Samuelson, and Monderer and Shapley, were right in suspecting that something quite interesting and deep was missing in our understanding of these economic theories - what was missing was the understanding of how the concept of fairness was intimately connected with all this. We showed that both potential and entropy stand for the concept of fairness in a distribution and that this is what the "invisible hand" is maximizing.

Building on this insight, we offer, in this paper, a novel interpretation of the Nash product and its connection with entropy and fairness. We show how both employ the same mathematical device to accomplish the same objective. Our earlier work showed the deep connection between potential game and statistical mechanics via the concept of entropy. In this paper, we show how these two frameworks are deeply connected with the NBS framework via the connection between entropy and the Nash product. Thus, all three puzzles - the "invisible 
hand," the potential, and the Nash product - are related in a deep and interesting manner to each other, and to entropy, and all these are all related to the same critical economic concept, namely, fairness.

\section{Potential Game Theoretic Framework: Summary of Past Work}

Before we can proceed, we need to recall the central ideas and results from our earlier work $[6,7]$, and so we summarize them here for the benefit of the reader.

Let us first recall the expression we derived for the utility of a rational agent employed in a company. We arrived at this by seeking to answer the basic question of why people seek employment. At the most fundamental, survival, level, it is to be able to pay bills now so that they can make a living, with the hope that the current job will lead to a better future. One hopes that the present job will lead to a better one next, acquired based on the experience from the current job, and to a series of better jobs in the future, and hence to a better life. Thus, the utility derived from a job is made up of two components: the immediate benefits of making a living (i.e., "present" utility) and the prospects of a better future life (i.e., "future" utility). There is, of course, the cost or disutility of effort or contribution to be accounted for as well.

Hence, we proposed that the effective utility from a job is determined by these three dominant elements: (i) utility from salary, (ii) disutility of effort or contribution, and (iii) utility from a fair opportunity for future prospects. By

effort, we do not mean just the physical effort alone, even though it is a part of it.

Thus, the effective utility for an agent is given by:

$$
h_{i}\left(S_{i}, N_{i}\right)=u_{i}-v_{i}+w_{i}
$$

where $h_{i}$ is the effective utility of an employee earning a salary $S_{i}$ by expending an appropriate effort, while competing with $\left(N_{i}-1\right)$ other agents in the same job category $i$ for a fair shot at a better future. $u(\cdot)$ is the utility derived from salary, 
$v(\cdot)$ the disutility from effort, and $w(\cdot)$ is the utility from a fair opportunity for a better future. Every agent tries to maximize its effective utility by picking an appropriate job category $i$.

\subsection{Utility of a Fair Opportunity for a Better Future}

The first two elements are rather straightforward to appreciate, but the third requires some discussion. Consider the following scenario. A group of freshly minted law school graduates (totaling $N_{i}$ ) have just been hired by a prestigious law firm as associates. They have been told that one of them will be promoted as partner in eight years depending on her or his performance. Let us say that the partnership is worth $\$ Q$. So any associate's chance of winning the coveted partnership goes as $1 / N_{i}$, where $N_{i}$ is the number of associates in her peer group $i$, her local competition. Therefore, her expected value for the award is $Q / N_{i}$, and the utility derived from it goes as $\ln \left(Q / N_{i}\right)$ because of diminishing marginal utility. Diminishing marginal utility is a well-known economics concept that states that the incremental benefit derived by consuming an additional unit of a resource decreases as one consumes more and more of it. This property is usually modeled by a logarithmic function. It is important to recognize here that the benefit $(\ln Q)$ lies in the future, but its cost or disutility $\left(\ln \left(1 / N_{i}\right)\right)$ is paid in the present, in the daily competition with one's peers towards the partnership. This is akin to buying a lottery ticket. The cost (say, \$1) of the ticket is incurred right away, right at the purchase, but the benefit of potentially winning a large sum lies in the future. For the time being, one is out $\$ 1$ - this disutility is to be accounted for right away.

Therefore, the disutility incurred towards a fair opportunity for career advancement in a competitive environment is:

$$
w_{i}\left(N_{i}\right)=-\gamma \ln N_{i}
$$

where $\gamma$ is a constant parameter. This equation models the effect of competitive interaction between agents. Considering the society at large, this equation captures the notion that in a fair society, an appropriately qualified agent with the 
necessary education, experience, and skills should have a fair shot at growth opportunities irrespective of her race, color, gender, and other such factors-i.e., it is a fair competitive environment. This is the cost or the disutility incurred for equality of access or equality of opportunity for a better life, for upward mobility. The category $i$ would correspond to her qualification category in the society. The other agents in that category are the ones she will be competing with for these growth opportunities.

\subsection{Modeling the Disutility of a Job}

For the utility derived from salary, we again employ the commonly used logarithmic utility function:

$$
u_{i}\left(S_{i}\right)=\alpha \ln S_{i}
$$

where $\alpha$ is another constant parameter. As for the effort component, every job has certain disutility associated with it. This disutility depends on a host of factors such as the investment in education needed to qualify oneself for the job, the experience to be acquired, working hours and schedule, quality of work, work environment, company culture, relocation anxieties, etc. To model this, one can combine $u$ and $v$ to compute

$$
u_{\text {net }}=a u-b v \quad(a \text { and } b \text { are positive constant parameters })
$$

which is the net utility (i.e., net benefit or gain) derived from a job after accounting for its cost. Typically, net utility will increase as $u$ increases (because of salary increase, for example). However, generally, after a point, the cost has increased so much, due to personal sacrifices such as working overtime, missing quality time with family, giving up on hobbies, job stress resulting in poor mental and physical health, etc., $u_{\text {net }}$ begins to decrease after reaching a maximum. The simplest model of this commonly occurring inverted-U profile is a quadratic function, as in

$$
u_{\text {net }}=a u-b u^{2} .
$$


Since, $u \sim \ln ($ Salary $)$, we get equation (6):

$$
v_{i}\left(S_{i}\right)=\beta\left(\ln S_{i}\right)^{2} .
$$

\subsection{Effective Utility from a Job}

Combining all three, we have

$$
h_{i}\left(S_{i}, N_{i}\right)=\alpha \ln S_{i}-\beta\left(\ln S_{i}\right)^{2}-\gamma \ln N_{i}
$$

where $\alpha, \beta, \gamma>0$.

In general, $\alpha, \beta$ and $\gamma$, which model the relative importance an agent assigns to these three elements, can vary from agent to agent. However, we examined the ideal situation where all agents have the same preferences and hence treat these as constant parameters.

\subsection{Equilibrium Income Distribution}

We then used the potential game theoretic framework to prove $[6,7]$ that a large population of agents with this utility function will reach Nash equilibrium. In potential games $[14,15,16]$, there exists a single scalar-valued global function, called a potential, that captures the necessary information about utilities. The

gradient of the potential is the utility. For such games, Nash equilibrium is reached when the potential is maximized.

So, using the potential game formalism, we have an employee's utility as the gradient of potential $\phi(\boldsymbol{x})$, i.e.,

$$
h_{i}(\boldsymbol{x}) \equiv \frac{\partial \phi(\boldsymbol{x})}{\partial x_{i}}
$$

where $x_{i}=N_{i} / N$ denotes the fraction of population at category $i$ and $\boldsymbol{x}$ is the population vector. Therefore, by integration (we replace partial derivative with total derivative because $h_{i}(\boldsymbol{x})$ can be reduced to $h_{i}\left(x_{i}\right)$ expressed in (1)-(6)),

$$
\phi(\boldsymbol{x})=\sum_{i=1}^{n} \int h_{i}(\boldsymbol{x}) \mathrm{d} x_{i} .
$$


We observe, using (7), that our game is a potential game with the potential function

$$
\phi(\boldsymbol{x})=\phi_{u}+\phi_{v}+\phi_{w}+\text { constant }
$$

where

$$
\begin{aligned}
\phi_{u} & =\alpha \sum_{i=1}^{n} x_{i} \ln S_{i} \\
\phi_{v} & =-\beta \sum_{i=1}^{n} x_{i}\left(\ln S_{i}\right)^{2} \\
\phi_{w} & =\frac{\gamma}{N} \ln \frac{N !}{\prod_{i=1}^{n}\left(N x_{i}\right) !}
\end{aligned}
$$

where we have used Stirling's approximation in equation (13).

One can see that $\phi(\boldsymbol{x})$ is strictly concave:

$$
\frac{\partial^{2} \phi(\boldsymbol{x})}{\partial x_{i}^{2}}=-\frac{\gamma}{x_{i}}<0
$$

Therefore, a unique Nash equilibrium for this game exists, where $\phi(\boldsymbol{x})$ is maximized, as per the well-known theorem in potential games [15, p. 60].

Thus, the self-organizing free market dynamics, where employees switch jobs, and companies switch employees, in search of better utilities or profits, ultimately reaches an equilibrium state, with an equilibrium income distribution. This happens when the potential $\phi(\boldsymbol{x})$ is maximized. The equilibrium income distribution is the following lognormal distribution:

$$
x_{i}=\frac{1}{S_{i} D} \exp \left[-\frac{\left(\ln S_{i}-\frac{\alpha+\gamma}{2 \beta}\right)^{2}}{\gamma / \beta}\right]
$$

where $D=N \exp \left[\lambda / \gamma-(\alpha+\gamma)^{2} / 4 \beta \gamma\right]$ and $\lambda$ is the Lagrange multiplier used in maximizing $\phi(\boldsymbol{x})$ with the $\sum_{i=1}^{n} x_{i}=1$ constraint.

We also proved that the effective utility, $h^{*}$, at equilibrium is given by:

$$
h^{*}=\gamma \ln Z-\gamma \ln N
$$

where $Z=\sum_{j=1}^{n} \exp \left(\left[\alpha \ln S_{j}-\beta\left(\ln S_{j}\right)^{2}\right] / \gamma\right)$ resembles the partition function seen in statistical mechanics (it is easy to show that $\lambda=h^{*}$ ). At equilibrium, 
all agents enjoy the same effective utility or effective welfare, $h^{*}$. Everyone is not making the same salary, of course, but they all enjoy the same effective utility. This is an important result, for it proves that all agents are treated equally with respect to the economic outcome, namely, effective utility. This proves that the ideal free-market exhibits outcome fairness.

We also proved that this distribution is socially optimal. A socially optimal distribution is one where the effective utility of the entire population (i.e., the total effective utility of society, $H$ ) is maximized. This is the outcome desired by utilitarians such as Jeremy Bentham and John Stuart Mill. This maximum total effective utility $\left(H^{*}\right)$ is given by

$$
H^{*}=\sum_{i=1}^{n} N_{i} h_{i}^{*}=\sum_{j=1}^{N} h_{j}^{*}=N h^{*}
$$

(since $h_{i}^{*}=h_{j}^{*}=h^{*}$ at equilibrium), subject to the constraints

$$
\begin{aligned}
\sum_{i=1}^{n} N_{i} S_{i} & =M \\
\sum_{i=1}^{n} N_{i} & =N
\end{aligned}
$$

Note that the index $i$ covers the $n$ different salary levels $S_{i}$, whereas $j$ covers the $N$ employees. $M$ is the total salary budget and $N$ is the total number of employees in a company.

Now that we have covered the necessary background work, we are ready to forge ahead to develop the Nash bargaining solution framework and its connection to maximum entropy. Towards that, let us first provide an intuitive explanation of the maximum entropy principle.

\section{Entropy as a Measure of Fairness: An Intuitive Explanation of}

$$
S=-\sum_{i=1}^{n} p_{i} \ln p_{i}
$$

As we know, entropy is maximized at statistical equilibrium. We also know that the equivalent defining criterion for statistical equilibrium is the equality 
of all accessible phase space cells, which, in fact, is the fundamental postulate of statistical mechanics. In other words, a given molecule is equally likely to be found in any one of the accessible phase space cells, at any given time.

For example, consider a very large number of identical gas molecules enclosed in a chamber. To say that a gas molecule, in the long run, is more likely to be found in the left half of the chamber than the right, and assign it a higher probability (say, $p($ left $)=0.6$ and $p$ (right) $=0.4$ ), would be a biased and an unfair assignment of probabilities. This assignment presumes some information that has not been given. What does one know about the molecule, or its surrounding space, that would make one prefer the left chamber over the right? There is no basis for this preference. The unequal assignment of probabilities is thus arbitrary and unwarranted. Therefore, the fairest assignment of probabilities is one of equality of outcomes, i.e., $p$ (left) $=0.5$ and $p$ (right) $=0.5$.

Let us examine this example further. Let us say that the chamber is divided into $n$ imaginary partitions of equal volume such that the molecules are free to move about from partition to partition. As noted, the essence of statistical equilibrium is the equality of all accessible phase space cells. But how is this connected to the maximization of entropy as defined by $S=-\sum_{i=1}^{n} p_{i} \ln p_{i}$ (not to be confused with salary $S)$ ? The connection is not obvious from this equation. However, there is a fascinating connection with an important implication on the true nature of entropy, as well as for the Nash product.

Now, the criterion for equilibrium in our chamber example is

$$
p_{1}=p_{2}=\ldots=p_{n}=p
$$

where $p_{i}$ is the probability of finding a given molecule in the partition $i$.

But how do we recognize and enforce this criterion in practice?

This is easy to do, for example, if there are only a few partitions in phase space, e.g., two partitions, left and right, as we did above. We would immediately recognize a non-equilibrium situation if one had $p$ (left) $=0.6$ and $p($ right $)=0.4$. And we would also recognize with equal ease the equilibrium situation, $p($ left $)=0.5$ and $p($ right $)=0.5$. 
But this gets trickier when there are a large number of partitions or cells in phase space. For example, what if there are 1000 partitions, as opposed to just two, and we are given the following two situations to evaluate?

$$
\begin{gathered}
p_{1}=0.0015, p_{2}=0.001, p_{3}=0.0009, \ldots, p_{1000}=0.0011 \\
p_{1}=0.0011, p_{2}=0.0008, p_{3}=0.0012, \ldots, p_{1000}=0.0009
\end{gathered}
$$

Since all the partitions are of equal volumes, at equilibrium,

$$
p_{1}=p_{2}=\ldots=p_{1000}=0.001
$$

How do we compare these two configurations and determine which one is closer to equilibrium? In general, how do we make this comparison among billions of such alternative configurations? How do we enforce this equality criterion?

One could compute the sum of squared residuals for comparison, or other such methods, but this gets to be really tedious and messy, when the number of molecules and the number of configurations run into billions as is typical in statistical mechanics. Is there a simpler, more elegant way of accomplishing this? It turns out there is, one that exploits a wonderful result regarding the product of a set of positive numbers that sum to a fixed amount. This is what is at the heart of the entropy equation, and the Nash product as we show later.

While we recognize the equilibrium state by the maximum entropy criterion, hidden in its mathematical form is the equality of all accessible cells criterion given by equation (20). To see this, we first observe that maximizing $\left\{-\sum_{i=1}^{n} p_{i} \ln p_{i}\right\}$ is the same as maximizing $\left\{-\ln \prod_{i=1}^{n} p_{i}^{p_{i}}\right\}$. And this is the same as minimizing $\left\{\ln \prod_{i=1}^{n} p_{i}^{p_{i}}\right\}$, which is the same as minimizing $\left\{\prod_{i=1}^{n} p_{i}^{p_{i}}\right\}$. Now, according to two well-known results (which are related), the arithmetic mean-geometric mean inequality theorem (AM-GM theorem) [17], and the Jensen's inequality, this product is minimized if and only if

$$
p_{1}=p_{2}=\ldots .=p_{n}
$$

where $0<p_{i}<1$ and $\sum_{i=1}^{n} p_{i}=1$ (one can also demonstrate this using the Lagrangian multiplier method, but, we believe, the geometric intuition is more 
transparent using the AM-GM theorem or the Jensen's inequality). This equality is, of course, the fundamental criterion for statistical equilibrium. Therefore, when we maximize entropy, what we are really doing is enforcing this equality constraint buried in it. This is a clever mathematical trick for enforcing equality, by exploiting this important relationship between the product and the sum of a set of positive numbers. Therefore, enforcing equality and hence fairness, is the objective buried in the mathematical trick of maximizing the product, $\left\{-\prod_{i=1}^{n} p_{i}^{p_{i}}\right\}$, which is the same as minimizing $\left\{\prod_{i=1}^{n} p_{i}^{p_{i}}\right\}$. We will now see in the next section how the same clever mathematical device of maximizing a product to test and enforce equality is employed for the Nash product.

We can see clearly now how entropy maximization is related to enforcing equality, which is the same as enforcing fairness - treating equal things equally. This insight that entropy really is a measure of fairness in a distribution has never been clearly recognized in statistical mechanics, in information theory, or in economics literature until the first author discussed its importance in his 2009-2010 papers [4, 5]. It is a historical accident that the concept of entropy was first discovered in the context of thermodynamics and, therefore, it has generally been identified as a measure of randomness or disorder. However, the true essence of entropy is fairness, which appears with different masks in different contexts (for a detailed discussion of this, see[7]).

\section{Nash Bargaining Solution}

We now derive the Nash bargaining solution to our problem. Following Nash's original formulation [8] for the two-player bargaining problem there has been extensive literature on this topic, which has been generalized to the $n$ -

player case using both cooperative and noncooperative approaches with many applications (we cite a few select papers here $[18,19,20,21,22,23,9,24,25$, $26,27])$.

We consider $N$ players labeled $j=1, \ldots, N$ (use $j$ instead of $i$ for the index of individual players), and we consider only the grand coalition involving all $N$ 
players because all $N$ players are required for the company to succeed. The surplus that this coalition generates is non-negative. Once this team forms, the game stops.

Suppose that players $1, \ldots, N$, have complementary skills which can be combined to produce a divisible pie of some total utility. The pie is produced and divided among the $N$ players only when all players reach an agreement on their shares $\left(h_{1}, h_{2}, \ldots, h_{N}\right)$, where $h_{j}$ is the share or the utility of the $j$ th player.

The following description of the NBS is adapted from [21]:

Consider a cooperative game of $N$ players (e.g., employees in a company). Let each individual player $j$ have an utility function $h_{j}(\boldsymbol{x}): \mathbb{X} \rightarrow \mathbb{R}$ where $\mathbb{X}$ is a convex, closed, and bounded set of $\mathbb{R}^{N}$. For example, in communication networks $\mathbb{X}$ denotes the space of throughputs. Let $\boldsymbol{h}_{\mathrm{d}}=\left[h_{\mathrm{d}, 1}, h_{\mathrm{d}, 2}, \ldots, h_{\mathrm{d}, N}\right]$ where $h_{\mathrm{d}, j}=h_{j}\left(\boldsymbol{x}_{\mathrm{d}}\right)$ for some $\boldsymbol{x}_{\mathrm{d}} \in \mathbb{X}$ denote a disagreement point which all the players agree to as a starting point for the game. In general, $\boldsymbol{h}_{\mathrm{d}}$ can be thought of as the vector of individual default utilities which the users would like to achieve, at least, if they enter the game. It is also referred to as the threat point. Let $\left[\mathbb{H}, \boldsymbol{h}_{\mathrm{d}}\right]$ denote the game defined on $\mathbb{X}$ with initial disagreement point $\boldsymbol{h}_{\mathrm{d}}$ where $\mathbb{H}$ denotes the image of the set $\mathbb{X}$ under $h(\cdot)$, i.e., $h(\mathbb{X})=\mathbb{H}$. Let $F\left[\cdot, \boldsymbol{h}_{\mathrm{d}}\right]: \mathbb{H} \rightarrow \mathbb{H}$ be an arbitration strategy. Then $F$ is said to be a NBS if it satisfies the four axioms below.

1. Let $\psi(\boldsymbol{h})=\boldsymbol{h}^{\prime}$ where $h_{j}^{\prime}=a_{j} h_{j}+b_{j}$ for $j=1,2, \ldots, N$ and $a_{j}>0, b_{j}$ are arbitrary constants. Then

$$
F\left[\psi(\mathbb{H}), \psi\left(\boldsymbol{h}_{\mathrm{d}}\right)\right]=\psi\left(F\left[\mathbb{H}, \boldsymbol{h}_{\mathrm{d}}\right]\right) .
$$

This states that the operating point in the space of strategies is invariant with respect to linear utility transformations.

2. The solution must satisfy

$$
\left(F\left[\mathbb{H}, \boldsymbol{h}_{\mathrm{d}}\right]\right)_{j} \geq h_{\mathrm{d}, j} \quad(j=1,2, \ldots, N)
$$


and furthermore there exists no $\boldsymbol{h} \in \mathbb{H}$ such that $h_{j} \geq\left(F\left[\mathbb{H}, \boldsymbol{h}_{\mathrm{d}}\right]\right)_{j}$ for all $j=1,2, \ldots, N$. This is the notion of Pareto optimality of the solution.

3. Let $\left[\mathbb{H}_{1}, \boldsymbol{h}_{\mathrm{d}}\right]$ and $\left[\mathbb{H}_{2}, \boldsymbol{h}_{\mathrm{d}}\right]$ be two games with the same initial agreement point such that:

$$
\begin{aligned}
\mathbb{H}_{1} & \subset \mathbb{H}_{2} \\
F\left[\mathbb{H}_{2}, \boldsymbol{h}_{\mathrm{d}}\right] & \in \mathbb{H}_{1} .
\end{aligned}
$$

Then $F\left[\mathbb{H}_{1}, \boldsymbol{h}_{\mathrm{d}}\right]=F\left[\mathbb{H}_{2}, \boldsymbol{h}_{\mathrm{d}}\right]$. This is called the independence of irrelevant alternatives axiom. This states that the NBS of a game with a larger set of strategies is the same as that of the smaller game if the arbitration point is a valid point for the smaller game. The additional strategies are superfluous.

4. Let $\mathbb{H}$ be symmetrical with respect to a subset $\mathbb{J} \subseteq\{1,2, \ldots, N\}$ of indices (i.e., let $j, k \in \mathbb{J}$ and $j<k$ ), then

$$
\left\{h_{1}, h_{2}, \ldots, h_{j-1}, h_{k}, h_{j+1}, \ldots, h_{k-1}, h_{j}, h_{k+1}, \ldots, h_{N}\right\} \in \mathbb{H} .
$$

If $h_{\mathrm{d}, j}=h_{\mathrm{d}, k}$, then $\left(F\left[\mathbb{H}, \boldsymbol{h}_{\mathrm{d}}\right]\right)_{j}=\left(F\left[\mathbb{H}, \boldsymbol{h}_{\mathrm{d}}\right]\right)_{k}$ for $j, k \in \mathbb{J}$. This is the axiom of symmetry which states that if the set of utilities is symmetric then, for any two players, if the initial agreement point corresponds to equal performance, then their arbitrated values are equal.

Nash proved that the unique solution, i.e., the NBS, of the game that satisfies the four axioms is given by the point which maximizes the expression $\prod_{j=1}^{N}\left(h_{j}(\boldsymbol{x})-\right.$ $\left.h_{\mathrm{d}, j}\right)$, known as the Nash product. This can be written as

$$
\max \prod_{j=1}^{N}\left(h_{j}(\boldsymbol{x})-h_{\mathrm{d}, j}\right)=\max \prod_{j=1}^{N}\left(h_{j}-h_{\mathrm{d}, j}\right)
$$

where $h_{j}$ denotes both the $j$ th player's utility function and its utility. 


\section{NBS of the Income Distribution Game}

In this section, we define the set of feasible utilities of the income game and show that it is a convex set. By maximizing the product of utilities over such set, we obtain the NBS of the income game and it is identical to the Nash equilibrium solution we had reported earlier [6].

Recall the utility function $h_{i}\left(S_{i}, N_{i}\right)$ defined in (7). Suppose the $n$ salary levels are predetermined. Then the utility for agents at each salary level solely depends on its occupancy, that is

$$
h_{i}\left(N_{i}\right)=h_{0, i}-\gamma \ln N_{i}
$$

where $h_{0, i} \equiv \alpha \ln S_{i}-\beta\left(\ln S_{i}\right)^{2}$ is a constant unique to the $i$ th salary level.

We now define the set of population $\mathbb{N}$

$$
\mathbb{N} \equiv\left\{N_{i} \in \mathbb{Z}_{+}: \sum_{i=1}^{n} N_{i} \leq N\right\}
$$

where the total number of agents does not exceed $N$. It is easy to verify that $\mathbb{N}$ is convex. Next, we construct the set of utilities $\mathbb{H}$ through the following mapping:

$$
\mathbb{H} \equiv\left\{h_{i}=h_{0, i}-\gamma \ln N_{i}: \sum_{i=1}^{n} N_{i} \leq N\right\}
$$

To show that $\mathbb{H}$ is also convex, let $\boldsymbol{h}_{1}=\boldsymbol{h}_{0}-\gamma \ln \boldsymbol{N}_{1}$ and $\boldsymbol{h}_{2}=\boldsymbol{h}_{0}-\gamma \ln \boldsymbol{N}_{2}$ be any two vectors of utilities in $\mathbb{H}$. They corresponds to two vectors of occupancies $\boldsymbol{N}_{1} \in \mathbb{N}$ and $\boldsymbol{N}_{2} \in \mathbb{N}$. Define $\boldsymbol{h}_{3}$ as the convex combination of $\boldsymbol{h}_{1}$ and $\boldsymbol{h}_{2}$ :

$$
\begin{aligned}
\boldsymbol{h}_{3} & \equiv t \boldsymbol{h}_{1}+(1-t) \boldsymbol{h}_{2} \\
& =\boldsymbol{h}_{0}-\gamma\left[t \ln \boldsymbol{N}_{1}+(1-t) \ln \boldsymbol{N}_{2}\right] \\
& =\boldsymbol{h}_{0}-\gamma \ln \boldsymbol{N}_{3}
\end{aligned}
$$

where $0 \leq t \leq 1$ is the ratio of the convex combination and $\boldsymbol{N}_{3} \equiv e^{t \ln \boldsymbol{N}_{1}+(1-t) \ln \boldsymbol{N}_{2}}$. 
We have

$$
\begin{aligned}
\sum_{i=1}^{n} N_{i, 3} & =\sum_{i=1}^{n} e^{t \ln N_{i, 1}+(1-t) \ln N_{i, 2}} \\
& \leq \sum_{i=1}^{n}\left[t e^{\ln N_{i, 1}}+(1-t) e^{\ln N_{i, 2}}\right] \\
& =\sum_{i=1}^{n}\left[t N_{i, 1}+(1-t) N_{i, 2}\right] \\
& =t \sum_{i=1}^{n} N_{i, 1}+(1-t) \sum_{i=1}^{n} N_{i, 2} \\
& \leq t N+(1-t) N \\
& =N
\end{aligned}
$$

where $N_{i, j}$ is the $i$ th component of the vector $\boldsymbol{N}_{j}$. The first inequality

$$
e^{t \ln N_{i, 1}+(1-t) \ln N_{i, 2}} \leq t e^{\ln N_{i, 1}}+(1-t) e^{\ln N_{i, 2}}
$$

follows Jensen's inequality where

$$
f\left(t x_{1}+(1-t) x_{2}\right) \leq t f\left(x_{1}\right)+(1-t) f\left(x_{2}\right)
$$

if the function $f(x)$ is convex; and the second follows the definition of $\mathbb{N}$. $\boldsymbol{h}_{3}$ is therefore also in $\mathbb{H}$ because $N_{3}$ is in $\mathbb{N}$.

Recall that the NBS is obtained by maximizing the Nash product in (26). By grouping players with the same amount of utility together (i.e., they are in the same income level), we convert the original NBS problem to the following problem:

$$
\max _{\boldsymbol{x} \in \mathbb{X}} \prod_{j=1}^{N}\left(h_{j}(\boldsymbol{x})-h_{\mathrm{d}, j}\right)=\max _{\boldsymbol{h} \in \mathbb{H}} \prod_{i=1}^{n} h_{i}^{N_{i}}
$$

where we have set the disagreement point or the threat point of the $j$ th player $h_{\mathrm{d}, j}$ to be zero (i.e., a player agrees to enter the game as long as her effective utility is greater than zero). Note that the first product is over all the players $(N)$ whereas the second is over all the levels $(n)$. 
Therefore, (34) is equivalent to solving

$$
\begin{array}{ll}
\max & g(\boldsymbol{N})=\sum_{i=1}^{n} N_{i} \ln h_{i} \\
\text { s.t. } & l(\boldsymbol{N})=\sum_{i=1}^{n} N_{i}-N \leq 0
\end{array}
$$

because logarithm is continuous and monotonic. The Karush-Kuhn-Tucker (KKT) necessary conditions of (35) are as follows:

$$
\begin{aligned}
\nabla g\left(\boldsymbol{N}^{*}\right) & =\mu \nabla l\left(\boldsymbol{N}^{*}\right) \\
l\left(\boldsymbol{N}^{*}\right) & \leq 0 \\
\mu & \geq 0 \\
\mu l\left(\boldsymbol{N}^{*}\right) & =0
\end{aligned}
$$

where $\mu$ is a KKT multiplier. Expanding (36), we have

$$
\ln h_{i}^{*}-\frac{\gamma}{h_{i}^{*}}=\mu \quad(i=1, \ldots, n) .
$$

There exists a unique solution to (40) where $h_{i}^{*}=h^{*}=\gamma / W\left(\gamma e^{-\mu}\right)$ and $W$ denotes the Lambert- $W$ (product $\log$ ) function. Since $W(x)$ monotonically increases as $x \geq 0$ increases, $h^{*}$ is maximized when $\mu>0$. From the complementary slackness in (39), we have $l\left(\boldsymbol{N}^{*}\right)=0$ or

$$
\sum_{i=1}^{n} N_{i}=N
$$

From (27), we have

$$
h^{*}=\alpha \ln S_{i}-\beta\left(\ln S_{i}\right)^{2}-\gamma \ln N_{i}^{*}
$$

The NBS of the income game therefore requires

$$
N_{i}^{*}=\frac{N}{S_{i} D} \exp \left[-\frac{\left(\ln S_{i}-\frac{\alpha+\gamma}{2 \beta}\right)^{2}}{\gamma / \beta}\right]
$$

where $D=\exp \left[h^{*} / \gamma-(\alpha+\gamma)^{2} / 4 \beta \gamma\right]$. Equation (43) is identical to the Nash equilibrium solution (15) since $\lambda=h^{*}$. This corresponds to all agents enjoying the same effective utility $h^{*}$ at equilibrium given by equation (16). 


\section{Summary and Conclusions}

We have presented a Nash bargaining solution to the problem of fair income distribution in an ideal free-market economy. As noted, this is the first time the NBS formalism has been proposed for this problem even though the formalism itself has been well-known for about seven decades and the fair inequality question has been open for over two centuries.

In addition, since the NBS outcome results in a lognormal distribution, which we have proved in our earlier work as the fairest outcome, we see the connection between NBS and fairness. By maximizing the Nash product, we are indeed maximizing fairness. We also showed that by maximizing entropy one was essentially maximizing fairness in the probability distribution by enforcing the equality of all accessible cells through the mathematical device of maximizing the product $-\ln \prod_{i=1}^{n} p_{i}^{p_{i}}$, which is the same as minimizing $\prod_{i=1}^{n} p_{i}^{p_{i}}$, by exploiting the arithmetic mean-geometric mean inequality theorem or Jensen's inequality.

In a similar manner, in NBS one again invokes the mathematical device of maximizing a product, this time the Nash product. Both techniques achieve the same desired result - the enforcement of equality. In the case of maximum entropy, we achieve the equality of the probabilities of all accessible phase space cells $-p_{1}=p_{2}=\ldots=p_{n}$. That is, we achieve maximizing fairness at equilibrium. Similarly for NBS, we achieve the equality of effective utilities for all agents $-h_{1}=h_{2}=\ldots=h_{N}=h^{*}$. That is, we achieve maximizing fairness at equilibrium.

Thus, the true economic objective of maximizing the Nash product is to treat all agents fairly subject to the Pareto optimality constraint. Since the fairness objective is buried deeply in the mathematical device of maximizing the product, just as it is buried in the formulation of maximum entropy principle, the fairness property is not obvious even when you probe it. So, naturally, people are surprised when they find the fairness outcome in the final results. That is why we have economists somewhat mystified, making observations such as "Nash product seems to have escaped up to now a meaningful interpretation," 
"Although the maximization of a product of utilities is a simple mathematical operation it lacks a straightforward interpretation; we view it simply as a technical device," and "The Unreasonable Fairness of Maximum Nash Welfare" [28], as quoted earlier. Thus, our work reveals the deep and surprising connection between the Nash product, entropy, and fairness. Achieving maximum fairness is the purpose of the maximum entropy principle as well as for the maximum Nash welfare function. Enforcing equality and hence fairness, under the given constraints, is the true objective buried in the mathematical device of maximizing a product-we see this in entropy and in Nash product.

\section{Acknowledgement}

This work is supported in part by the Center for the Management of Systemic Risk (CMSR) at Columbia University. The authors thank Resmi Suresh for her helpful comments and assistance with the preparation of this manuscript.

\section{References}

[1] T. Piketty, A. Goldhammer, Capital in the twenty-first century, Belknap Press, 2014.

[2] J. E. Stiglitz, The Great Divide: Unequal Societies and What We Can Do about Them, W. W. Norton \& Company, 2015.

[3] R. B. Reich, Saving Capitalism: For the Many, Not the Few, Knopf, 2015.

[4] V. Venkatasubramanian, What is fair pay for executives? an information theoretic analysis of wage distributions, Entropy 11 (4) (2009) 766-781.

[5] V. Venkatasubramanian, Fairness is an emergent self-organized property of the free market for labor, Entropy 12 (6) (2010) 1514-1531.

[6] V. Venkatasubramanian, Y. Luo, J. Sethuraman, How much inequality in income is fair? a microeconomic game theoretic perspective, Physica A: Statistical Mechanics and its Applications 435 (2015) 120-138. 
[7] V. Venkatasubramanian, How Much Inequality is Fair? Mathematical Principles of a Moral, Optimal, and Stable Capitalist Society, Columbia University Press, 2017.

[8] J. F. Nash, The bargaining problem, Econometrica 18 (2) (1950) 155-162.

[9] A. Muthoo, Bargaining Theory with Applications, Cambridge University Press, UK, 1999.

[10] W. Trockel, On the meaning of the nash product, Tech. rep., Bielefed: IMW-working paper Nr. 354 (2003).

[11] M. J. Osborne, A. Rubinstein, A Course in Game Theory, MIT Press, Cambridge, MA, 1994.

[12] D. Monderer, L. S. Shapley, Potential games, Games and economic behavior 14 (1) (1996) 124-143.

[13] P. A. Samuelson, Maximum principles in analytical economics, The American Economic Review (1972) 249-262.

[14] R. W. Rosenthal, A class of games possessing pure-strategy nash equilibria, International Journal of Game Theory 2 (1) (1973) 65-67.

[15] W. H. Sandholm, Population games and evolutionary dynamics, MIT press, 2010.

[16] D. Easley, J. Kleinberg, Networks, crowds, and markets: Reasoning about a highly connected world, Cambridge University Press, 2010.

[17] A. Lohwater, Introduction to inequalities, Tech. rep., e-book online (1982).

[18] J. Harsanyi, A simplified bargaining model for the n-person cooperative game, Int. Econ. Rev. 4 (1963) 194-220.

[19] A. Rubinstein, Perfect equilibrium in a bargaining model, Econometrica 50 (1982) 97-109. 
[20] A. R. K. G. Binmore, A. Wolinsky, The nash bargaining solution in economic modelling, RAND Journal of Economics 17 (1986) 176-188.

[21] L. M. R. R. Mazumdar, C. Douligeris, Fairness in network optimal flow control: Optimality of product forms, IEEE Trans. Commun. 39 (1991) $775-782$.

[22] D. R. K. Chatterjee, B. Dutta, K. Sengupta, A noncooperative theory of coalitional bargaining, Review of Economic Studies 60 (1993) 463-477.

[23] V. Krishna, R. Serrano, Multilateral bargaining, Review of Economic Studies 63 (1996) 61-80.

[24] R. R. M. H. Yaïche, C. Rosenberg, Game theoretic framework for bandwidth allocation and pricing in broadband networks, IEEE/ACM TRANSACTIONS ON NETWORKING, VOL. 8, NO. 5, OCTOBER 20008 (5) (2000) 667-678.

[25] D. Ray, A Game Theoretic Perspective on Coalition Formation, Oxford University Press, U.K., 2007.

[26] A. Okada, The nash bargaining solution in general n-person cooperative games, Journal of Economic Theory 6 (145) (2010) 2356-2379.

[27] O. Compte, P. Jehiel, The coalitional nash bargaining solution, Econometrica 78 (5) (2010) 1593-1623.

[28] I. Caragiannis, D. Kurokawa, H. Moulin, A. D. Procaccia, N. Shah, J. Wang, Ec'16, in: Proceedings of the 2016 ACM Conference on Economics and Computation, 2016, pp. 305-322. 